\title{
HUJJATLAR VA AXBOROT SO`ROVLARINI AVTOMATIK INDEKSATSIYA QILISH
}

Elov B.B.

Alisher Navoiy nomidagi Toshkent davlat

o'zbek tili va adabiyoti universiteti

Axborot texnologiyalari kafedrasi mudiri, t.f.n $\mathrm{PhD}$

Kayumov S.O'

Alisher Navoiy nomidagi Toshkent davlat

o'zbek tili va adabiyoti universiteti,

Kompyuter lingvistikasi mutaxassisligi 1-bosqich magistranti

Annotatsiya. Bugun biz demokratik jarayonlarni chuqurlashtirish, aholining siyosiy faolligini oshirish, fuqarolarning mamlakatimiz siyosiy va ijtimoiy hayotidagi amaliy ishtiroki haqida so ' $z$ yuritar ekanmiz, albatta, axborot erkinligini ta'minlamasdan, ommaviy axborot vositalarini odamlar $\mathrm{o}^{6} \mathrm{z}$ fikr va $\mathrm{g}^{\text {'oyalarini, }}$ sodir bo 'layotgan voqealarga $\mathrm{o}^{6} \mathrm{z}$ munosabati va pozitsiyasini erkin ifoda etadigan minbarga aylantirmasdan turib, bu maqsadlarga erishib bo'lmasligini o'zimizga yaxshi tasavvur etamiz.

Kalit so'zlar: ommaviy axborot vositalari, axborot sohasidagi huquq, demokratik jamiyat asoslari

Annotation. Today, when we talk about deepening the democratic process, increasing the political activity of the population, the practical participation of citizens in the political and social life of our country, of course, without ensuring freedom of information, the media we have a good idea that these goals cannot be achieved without turning their attitude and position into a platform for free expression of events.

Keywords: mass media, information law, basics of democratic society

Ma'lumki, fuqarolarning axborot sohasidagi huquq va erkinliklarini ta'minlash masalasi insonning axborot olish, axborotni va $\mathrm{o}^{\mathrm{x}} \mathrm{z}$ shaxsiy fikrini tarqatish huquqi va erkinligini o'zida mujassam etgan bo'lib, bu $\mathrm{O}^{\prime} z$ zekistonda demokratik jamiyat asoslarini barpo etishning muhim sharti, ta'bir joiz bo'lsa, tamal toshi hisoblanadi.

Mamlakatimizda o'tgan yillar davomida, ayniqsa oxirgi 10 yilda ommaviy axborot vositalarini yanada liberallashtirish, so' $z$ erkinligini ta'minlashga 
qaratilgan keng qamrovli tashkiliy-huquqiy chora-tadbirlar amalga oshirildi.

Yurtimizda avvalo ommaviy axborot vositalari rivojini ta'minlaydigan, demokratik talab va standartlarga to 'la mos keladigan mustahkam qonunchilik bazasi yaratildi. Ushbu davrda ommaviy axborot vositalarini erkin va jadal rivojlantirishga, axborot sohasining samarali faoliyat ko'rsatishini ta'minlashga qaratilgan 10 ga yaqin qonun hujjatlari qabul qilindi.

«Axborot erkinligi prinsiplari va kafolatlari to ' $g$ 'risida»gi Qonunning qabul qilinishi har kimning axborotni erkin va moneliksiz olish hamda foydalanish huquqlarini amalga oshirishda, shuningdek, axborotning muhofaza qilinishi, shaxs, jamiyat va davlatning axborot borasidagi xavfsizligini ta'minlashda muhim ahamiyat kasb etdi.

So"nggi yillarda yangi tahrirdagi «Ommaviy axborot vositalari to 'g'risida»gi Qonunga, shuningdek, «Telekommunikatsiyalar to'g'risida», «Reklama to 'g'risida», «Mualliflik huquqi va turdosh huquqlar to'g'risida»gi va boshqa bir qator qonunchilik hujjatlariga tegishli o'zgartish va qo'shimchalar kiritildi. Bu esa yangi siyosiy shart-sharoitlarda ommaviy axborot vositalari sohasidagi demokratik islohotlarni yanada chuqurlashtirishni ta'minladi. Shu bilan birga, nodavlat ommaviy axborot vositalarini rivojlantirish, ularning axborot sohasini demokratlashtirishda faol ishtirokini ta'minlashga qaratilgan keng qamrovli institutsional islohotlar amalga oshirildi.

Nodavlat ommaviy axborot vositalarini qo'llab-quvvatlash, ularning moddiy-texnik bazasi va kadrlar salohiyatini mustahkamlash maqsadida bir qancha jamoat tashkilotlari tashkil qilindi. O'z tarkibida 100 dan ortiq elektron ommaviy axborot vositasini birlashtirgan Nodavlat elektron ommaviy axborot vositalari milliy assotsiatsiyasi, O'zbekiston mustaqil bosma ommaviy axborot vositalari va axborot agentliklarini qo'llab-quvvatlash va rivojlantirish jamoat fondi shular jumlasidandir.

Yuridik va jismoniy shaxslarning, axborot texnologiyalari va tizimlarini qo' llagan holda, axborot resurslaridan foydalanish mexanizmlarini belgilab bergan yangi tahrirdagi «Axborotlashtirish to'g'risida»gi Qonunning qabul qilinishi ommaviy axborot vositalarini modernizatsiya qilishni jadal rivojlantirishda muhim ahamiyat kasb etdi.

\section{MATERIAL VA METODLAR}

O'zbekistonda keyingi yillarda sun'iy yo'ldosh aloqa tarmog'i orqali teleradiodasturlarni tarqatish yo'lga qo'yildi. Bugungi kunda mamlakatimiz telekommunikatsiyalar tizimi dunyoning 180 ta mamlakatiga 28 ta yo "nalish 
bo'yicha to 'g'ridan-to'g'ri chiqadigan xalqaro kanallarga ega. Yurtimizdagi teleradiokanallar tomonidan tayyorlanayotgan ko'rsatuv va eshittirishlar Internet global tarmog'i orqali real vaqt rejimida jahonga uzatilmoqda.

Dunyodagi etakchi mamlakatlar tajribasiga tayangan holda, kadrlar tayyorlash va qayta tayyorlash milliy tizimining takomillashtirilishi axborot sohasidagi faoliyatning sifati va saviyasini oshirishga qaratilgan chora-tadbirlar tizimida ko'p jihatdan hal qiluvchi ahamiyatga ega bo'ldi.

Bunday keng miqyosdagi ishlar natijasida faqat keyingi 10 yilning o'zida bosma ommaviy axborot vositalarining soni 1,5 barobar, elektron ommaviy axborot vositalarining soni esa 7 barobar ko'payib, bugungi kunda ularning umumiy soni qariyb 1200 taga etdi. Mavjud barcha telekanallarning qariyb 53 foizi, radiokanallarning esa 85 foizi nodavlat ommaviy axborot vositalari hisoblanadi. Ommaviy axborot vositalari $\mathrm{O}^{`}$ zbekistonda yashaydigan millat va elatlarning 7 ta tilida faoliyat olib boradi, shuningdek, bosma materiallar va teleko'rsatuvlar ingliz tilida ham tarqatilmoqda.

Yana bir muhim yangilik efir orqali uzatiladigan materiallarni tayyorlash jarayoniga zamonaviy raqamli va multimedia texnologiyalari joriy qilinayotganida namoyon bo'lmoqda. Yurtimizda Internet tizimidan foydalanuvchilar safi jadal sur'atlar bilan kengayib bormoqda. Bugungi kunda ularning soni 6 milliondan ortib ketgani ham buni tasdiqlab turibdi.

Mamlakatimizda fuqarolarning axborot sohasidagi huquq va erkinliklarini ta'minlash borasida amalga oshirilgan keng ko'lamli ishlarni tanqidiy baholar ekanmiz, muhim bir masalaga alohida e'tibor qaratishimiz zarur, deb o'ylayman. Ya'ni, bu o'rinda gap ommaviy axborot vositalari va davlat hokimiyati organlari o'rtasidagi munosabatlarning ustuvor jihatlarini to'g'ri belgilash, jumladan, ommaviy axborot vositalari faoliyati ustidan nazorat qilishning iqtisodiy mexanizmlarini, axborot manbalarining yopiqligini, shuningdek, tahririyatlarga hokimiyat organlari va ma'muriy tuzilmalar tomonidan bo'layotgan ma'lum darajadagi bosimlarni bartaraf qilish bilan bog'liq muammoli masalalarni hal etish haqida bormoqda.

Shu boisdan quyidagi chora-tadbirlarni amalga oshirish o'ta dolzarb ahamiyat kasb etmoqda:

Birinchi. «Davlat hokimiyati va boshqaruvi organlari faoliyatining ochiqligi to' $\mathrm{g}^{6}$ risida» Qonun qabul qilish zarur. $\mathrm{Bu} \mathrm{o}^{6} \mathrm{z}$ navbatida fuqarolarning axborot sohasidagi konstitutsiyaviy huquqini yanada kengroq amalga oshirish imkoniyatini yaratib berish bilan birga, davlat hokimiyati va boshqaruvi organlarining qabul 
qilinayotgan qarorlar sifatini oshirish borasidagi mas'uliyatini ham ko'p jihatdan kuchaytirgan bo'lur edi.

Ushbu qonun davlat hokimiyati organlari faoliyati haqida jamoatchilikni xabardor qilib borish tartiblarini aniq belgilab berishi, aholining, jamoat birlashmalarining davlat hokimiyati organlari tomonidan qabul qilinayotgan qarorlar, avvalambor fuqarolarning huquq va erkinliklari, qonuniy manfaatlari bilan bog'liq qarorlar haqidagi axborotlardan keng xabardor bo'lib borishini ta'minlashi lozim.

Mazkur qonunning joriy etilishi ijro hokimiyati organlari faoliyatining, yurtimizda amalga oshirilayotgan islohotlarning, davlatning ichki hamda tashqi siyosatining ochiqligi va oshkoraligini, mamlakatimiz va xorijda kechayotgan voqea-hodisalar xususida fikrlar xilma-xilligi va siyosiy plyuralizmni hisobga olgan holda, ta'minlashi zarur.

Ikkinchi. Demokratlashtirish jarayonlarida ahamiyati tobora ortib borayotgan axborot kommunikatsiyalari sohasining eng muhim tarmoqlaridan biri bo'lgan teleradio tizimini rivojlantirishga qaratilgan «Teleradioeshittirishlar to ' $\mathrm{g}$ 'risida» Qonun qabul qilish haqida taklif kiritilmoqda.

Ma'lumki, hozirgi vaqtda ushbu sohadagi faoliyat ommaviy axborot vositalari, radiochastota spektri, telekommunikatsiyalar, axborotlashtirish to ' $g$ 'risidagi qonunlarning muayyan moddalari bilan tartibga solib kelinmoqda. Shu bilan birga, teleradioeshittirishlar sohasini mustaqil qudratli industriya sifatida qayta tashkil etish, teleradioeshittirishlarning yangi shakl va turlarining paydo bo'lishi teleradiodasturlarni tayyorlash va uzatish jarayonida yuzaga keladigan munosabatlarni tizimli va keng ko' lamli ravishda tartibga soladigan yaxlit qonunni kuchga kiritish zaruratini tug'dirmoqda.

Ushbu qonunning qabul qilinishi teleradiodasturlarni tayyorlash va tarqatish sohasida raqobatni yanada kuchaytirish, teleradiodasturlarni uzatish borasida mobil va raqamli televideniye kabi ilg'or zamonaviy texnologiyalarni joriy etish, teleindustriyaning istiqbolli yangi tarmoqlarini tashkil qilish uchun zarur shartlarni yaratish imkonini beradi.

$\mathrm{Bu}$ qonun milliy teleradioeshittirishlar tizimi faoliyatini huquqiy tartibga solish mexanizmlarini va moliyalashtirish manbalarining erkinligi va mustaqilligi kabi prinsiplarini belgilab berishga, radiochastotalarni olish uchun o'tkaziladigan tanlovlarning demokratik va oshkoraligini ta'minlash, teng raqobat va elektron media-bozor tarmoqlarining monopollashuviga yo'1 qo'ymaslik uchun sharoit yaratish va boshqa bir qator muammolarni echishni nazarda tutadi. 
Uchinchi. Ommaviy axborot vositalari erkinligi va mustaqilligini yanada mustahkamlash mualliflik huquqlari va intellektual mulkni ishonchli himoya qilishning huquqiy kafolatlari va mexanizmlarini kuchaytirish, axborot sohasiga bozor mexanizmlarini joriy qilish bilan bevosita bog'liqdir.

Ushbu vazifani amalga oshirish uchun *«Ommaviy axborot vositalari faoliyatining iqtisodiy asoslari to ' $\mathrm{g}$ 'risida», «Ommaviy axborot vositalarini davlat tomonidan qo'llab-quvvatlash kafolatlari to'g'risida»* gi qonunlarni qabul qilish taklif etiladi. Bu qonunlar axborot bozori ishtirokchilari faoliyati samaradorligini kuchaytirish va ularning iqtisodiy manfaatlarini himoya qilish, qo'shimcha iqtisodiy preferensiyalar yaratish va milliy axborot makonini izchil rivojlantirishni ta'minlashga qaratilgan boshqa tashkiliy-huquqiy chora-tadbirlarni amalga oshirishni nazarda tutadi.

To'rtinchi. Ommaviy axborot vositalarining davlat hokimiyati va boshqaruv organlari faoliyati ustidan jamoatchilik va parlament nazoratini ta'minlash, hokimiyat va jamoatchilik o'rtasida mustahkam aloqa o'rnatish borasidagi rolini kuchaytirishga qaratilgan samarali huquqiy mexanizmlarni yaratish maqsadida *«Ommaviy axborot vositalari to 'g'risida», «Axborot erkinligi prinsiplari va kafolatlari to ' g'risida»* gi va boshqa bir qator qonun hujjatlariga tegishli o'zgartish va qo'shimchalar kiritish taklif etiladi.

Bunda, xususan, davlat va jamoat birlashmalari axborot xizmatlari, mediatuzilmalar ishini faollashtirish bo'yicha keng ko'lamli chora-tadbirlarni ko'zda tutish lozim. Ommaviy axborot vositalarining axborot olish yuzasidan murojaatlarini ko'rib chiqish muddatlarini qisqartirish, axborot olish sohasidagi qonunchilik talablarini buzganlik uchun yuridik va mansabdor shaxslarning ma'muriy javobgarligini kuchaytirish va boshqa chora-tadbirlarni ta'minlashga qaratilgan huquqiy mexanizmlar ustida ish olib borish zarur.

Shu bilan birga, siyosiy modernizatsiya jarayonlarida tobora muhim ahamiyat kasb etib borayotgan zamonaviy axborot-kommunikatsiya texnologiyalaridan davlat va jamiyat qurilishi tizimida keng foydalanish lozim. «Telekommunikatsiyalar to ' $g$ 'risida»gi Qonun normalarini yanada takomillashtirish, raqamli teleradioeshittirish tizimiga o'tish bo'yicha tadbirlar davlat dasturining ishlab chiqilishi ham ushbu vazifani hal etishga xizmat qiladi. Mazkur dastur raqamli teleradioeshittirishlar infratuzilmasini shakllantirish, bu boradagi faoliyatni huquqiy jihatdan tartibga soladigan samarali tizimni yaratishga qaratilishi darkor.

Muxtasar aytganda, yuqorida zikr etilgan chora-tadbirlarning amalga 
oshirilishi ommaviy axborot vositalarining fuqarolik jamiyati institutlari tizimidagi o'rni va rolini yanada mustahkamlashga, fuqarolarning so' $z$ erkinligi va tanlash erkinligini ta'minlashga qaratilgan konstitutsiyaviy huquqlarini yanada to'liq ro'yobga chiqarishga yordam beradi.

\section{Foydalanilgan adabiyotlar:}

1 A.K.Ibragimov. Catalog of training tasks for training special endurance of yong gril handboll players // Academical.An International Multidisciplenary Research Journal 2.35-39p

2. A.K.Ibragimov, F.I.Mo'minov. Methodology of optimization of trainingfor young players // International Journal For Innovative Engineering and Management Research Vol 10 Issue02, Feb2021 ISSN 2456 - 5083 Page 43-48.

3 R.R.Raxmonov. Distribution of training loads at the stage of competitive preparation for middle runners // ACADEMICIA: An International Multidisciplinary Research Journal https://saarj.com ISSN: 2249-7137 Vol. 11, Issue 2, February 2021 Impact Factor: SJIF $2021=7.492$ Pages 768-777. 